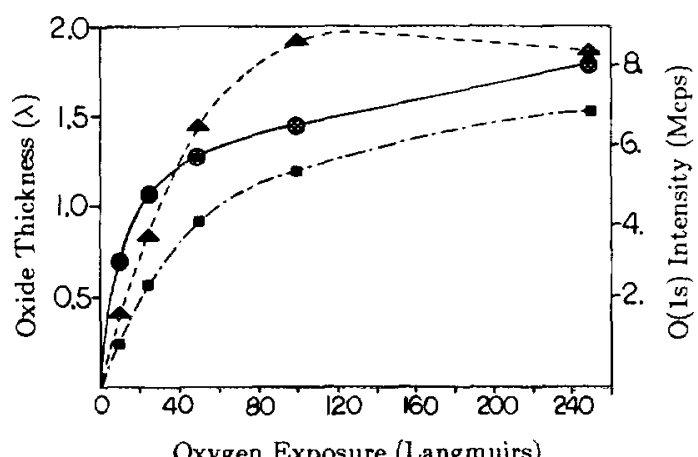

FIG. 2. Oxide film thickness results obtained using simple data reduction procedure (triangles) and the method presented here (squares). The $\mathrm{O}(1 s$ ) absolute signal increase (solid circles) is shown for comparison. Note different $y$-axis scale for the $O(1 s)$ results.

ation of the original Fe metal intensity upon oxidant exposure. This yields thickness values in units of $\lambda$ (escape depth). The results obtained from the method presented here are given in the lower trace (solid squares) and those obtained using integral background subtraction and Gaussian peak fit are given in the upper trace (triangles). The trace with the solid circles corresponds to the increase in $O(1 s)$ intensity for the same exposures (note the different $y$ axis scale on the right). The growth of the $O(1 s)$ clearly indicates that film growth continues throughout the exposure levels presented here. The use of simplistic data analysis for the $\mathrm{Fe}(2 p)$ region overestimates the contributions of the oxide peaks at the expense of the metallic peak in the early stages of oxidation.
'D. A. Shirley, Phys. Rev. B 5, 4709 (1972).

${ }^{2}$ S. Tougaard and B. Jorgensen, Surf. Sci. 143, 482 (1984)

'G. K. Wertheim and S. B. Dicenzo, Electron Spectrosc. Relat. Phenom. 37, 57 (1985)

${ }^{4}$ B. L. Maschhoff and N. R. Armstrong (in preparation).

\title{
Summary Abstract: Self-deconvolution in CVV Auger electron spectroscopy
}

\author{
A. G. B. M. Sasse, D. G. Lakerveld, and A. van Silfhout
}

University of Twente, Faculty of Applied Physics, P. O. Box 217,7500 AE. Enschede, The Netherlands

(Received 25 September 1987; accepted 7 December 1987)

Auger electron spectroscopy (AES) is now widely used as a technique to investigate solid surfaces. It gives information about elemental composition as well as the electronic states in the surface region, including information on the band structure of solids. ${ }^{1-4}$

Recently, several papers ${ }^{5-10}$ discussed how to obtain the density of states (DOS) from the measured Auger $C V V$ intensity $N(E)$. The measured Auger intensities appear as convolution products of the local transition densities in the valence band for the two electrons involved, distorted by an escape function and all kinds of loss mechanisms, such as plasmon losses, secondary background, and instrumental broadening. ${ }^{11,12}$ Assuming that an accurate correction of this distortion is feasible, we can describe $N(E)$ as a summation over all Auger transitions with the same final state energy, each weighted with the Auger matrix element $\left|M\left(E_{v}, \Delta\right)\right|^{2}$ of the respective Auger process, neglecting the screening of the $s$-like valence-band electrons of the core hole and the final state shakeoff effects ${ }^{10,13-17}$ :

$$
N\left(E_{v}\right)=\int\left|M\left(E_{v}, \Delta\right)\right|^{2} D\left(E_{v}-\Delta\right) D\left(E_{v}+\Delta\right) d \Delta .
$$

Due to the core-hole localization, it is possible to write the Auger matrix element $M\left(E_{v}, \Delta\right)$ in terms of atomic Auger matrix elements and of angular momentum components of the density of states localized at the same site as the core hole (LDOS).
Self-deconvolution of $N(E)$ with the assumption of constant matrix elements across the valence band ${ }^{13-17}$ can be interpreted as a transition density of states (TDOS). If the atomic matrix elements are known, then the TDOS can be interpreted as LDOS. ${ }^{13-15}$

To obtain fundamental information on the electrons involved in the Auger intensity spectrum $N(E)$ an accurate unfolding of the spectrum is needed. However, this unfolding appears to be quite troublesome and far from trivial.

Onsgaard et al ${ }^{18}$ suggested a new method to unfold a selfconvolution with the help of an iterative minimizing method. They tried to minimize a calculated convolution by constructing a trial unfold function with respect to the measured convolution. This method bypasses numerical problems such as the ambiguities of the square root of the complex phase in Fourier transforms $s^{8,15,16}$ and the difficulties of the choice of the starting point of the sequential digital method developed by Hagstrum and Becker. ${ }^{6,7}$

As an iterative minimizing method we used in this work a modified Levenerg-Marquardt method (LM) ${ }^{19}$ The LM method states that the direction of the search of the minimum lies somewhere between the Gauss-Newton method and the steepest descent method. This method combines the rapidity of the Gauss-Newton and the absolute convergence of the steepest descent method. To obtain a global approximation in the LM method the trial TDOS was composed of splines. ${ }^{20}$ Global approximation methods determine auto- 


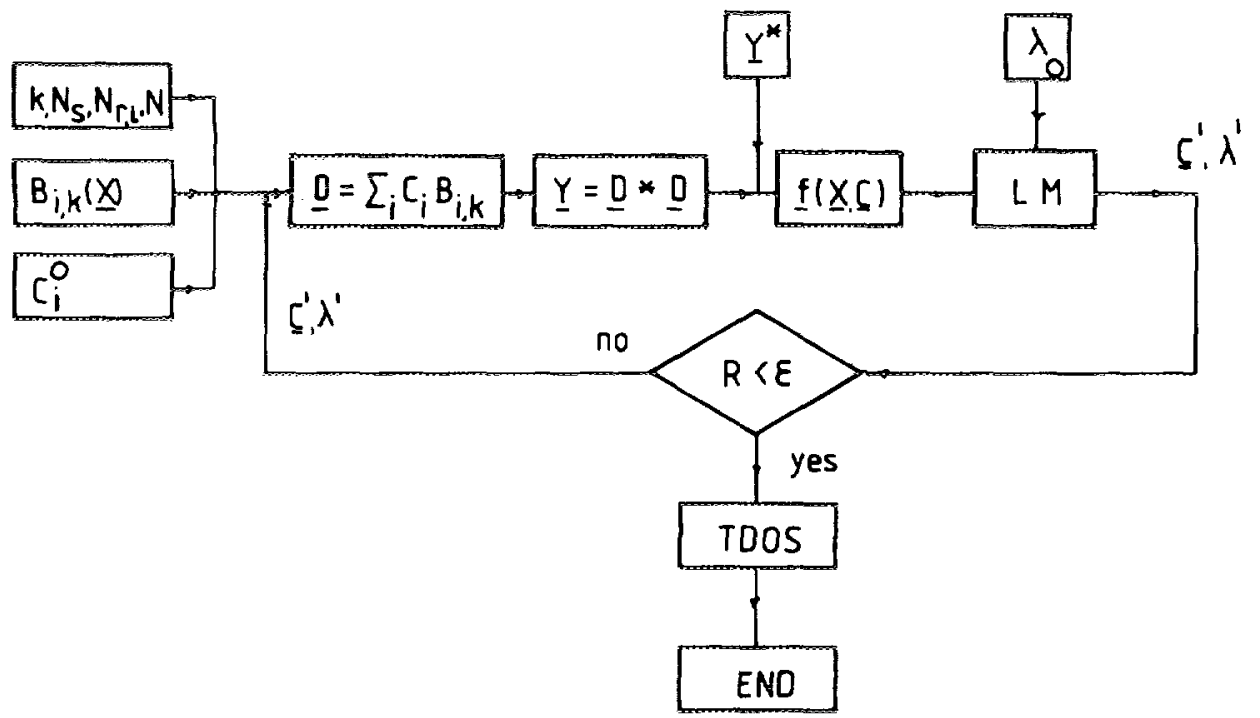

FIG. 1. The iteration scheme of the self-deconvolution calculation. $k=$ order of the spline; $N_{s}=$ number of splines; $N_{r}=$ num ber of boundary splines; $N=$ number of intervals; $\boldsymbol{B}_{i, k}(x)=$ spline of order $k$ starting on the ith interval; $C_{1}^{0}=$ initial choice of the spline coefficients $C_{i} ; \mathbf{f}(\mathbf{x}, \mathbf{c})=\mathbf{Y}-\mathbf{Y}^{*}$ $R=$ residue of the fit, which is defined as the square root of the sum of the squares of the difference between the seif-fold of the trial function and the original corrected data $N(E) ; \epsilon=$ constant; $\lambda_{0}=$ initial value of the Levenberg-Marquardt constant, which determines the ratio of the Gauss-Newton and the steepest descent minimizing routines.

matically the line regions over the entire spectrum, in contrast with local approximation methods using polynoms.

In this paper we have used more advanced types of splines, $B_{i, k}(x),{ }^{20}$ than applied by Onsgaard $e t a l$. These splines, defined on a subinterval $\left(x_{i}, x_{i+k}\right)$ of the interval $\left(x_{1}, x_{N}\right)$, can be constructed by a recursive sequence with variable order $k$ :

$$
\begin{aligned}
B_{i, k}(x)= & \frac{x-x_{i}}{x_{i+k-1}-x_{i}} B_{i, k-1}(x) \\
& +\frac{x_{i+k}-x}{x_{i+k}-x_{i+1}} B_{i+1 . k-1}(x),
\end{aligned}
$$

with

$$
B_{i, 1}(x)=\mid \begin{array}{ll}
1 ; x_{i} \leqslant x<x_{i+1} & k=2,3,4, \ldots \\
0 ; \text { elsewhere } & i=1, \ldots, N
\end{array} .
$$

Functions composed of splines of order $k$ and its derivatives are continuous to order of $k-1$ on the interval $\left(x_{1}, x_{N}\right)$.

The TDOS can be approximated by $N_{s}$ splines:

$$
D(x, \mathbf{c})=\sum_{i}^{N_{i}} c_{i, k} B_{i, k}(x)
$$

Using Eq. (2) and assuming constant matrix elements we can write:

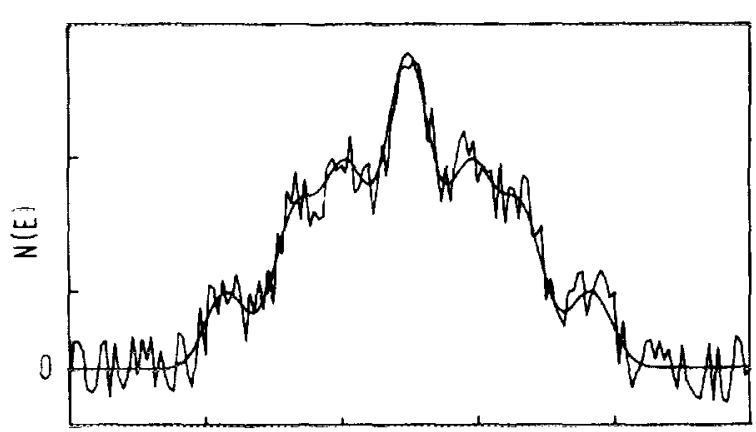

FIG. 2. The initial artificial data $[N(E)]$ (a) without noise, and (b) with $10 \%$ noise. These spectra are the input data of the self-deconvolution routine.

\footnotetext{
J. Vac. Scl. Technol. A, Vol. 6, No. 3, May/Jun 1988
}

$$
\begin{aligned}
Y(x, \mathrm{c}) & =\int D(x-\Delta) D(x+\Delta) d \Delta \\
& =\int \sum_{i} \sum_{j} c_{i, k} c_{j, k} B_{i, k}(x-\Delta) B_{j, k}(x+\Delta) d \Delta .
\end{aligned}
$$

The function $\mathbf{f}(\mathbf{x}, \mathbf{c})$ used in the LM can be defined as

$$
\begin{aligned}
& \mathbf{f}(\mathbf{x}, \mathbf{c})=\mathbf{Y}(\mathbf{x}, \mathbf{c})-\mathbf{Y}^{*}, \\
& F(\mathbf{x}, \mathbf{c})=\mathbf{f}^{T} \mathbf{f},
\end{aligned}
$$

where $\mathrm{Y}^{*}$ is the measured $N(E)$. The $\mathrm{LM}$ method minimizes the function $F(\mathbf{x}, \mathbf{c})$ and computes a set of $c_{i}$ 's. From this set we can construct the TDOS by applying Eq. (3). See Fig. 1.

The routine can be executed on a personal computer (Olivetti M24 with coprocessor). The calculation time per iteration depends mainly on the number of data points of $N(E)$ $(200$ points $=10 \mathrm{~s})$. On the average we need about 20 iterations to obtain a solution. This can be improved significantly by a better initial guess of the $c_{i}$ 's.

To give an indication of the reliability of the routine, two tests with artificial data are shown in Figs. 2 and 3. First, we

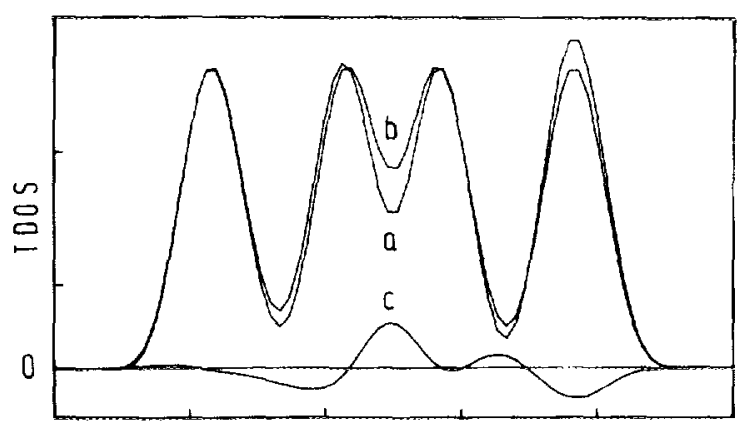

Fig. 3. The solutions of the unfolding of $N(E)$ : (a) $N(E)$ without noise, (b) $N(E)$ with $10 \%$ noise, and (c) the difference between the solution of (a) and (b). The smooth $N(E)$ is the self-convolution of a trial TDOS. The absolute error per data point between the initial TDOS and the calculated TDOS is smaller than $1.0 \times 10^{-7}$. This indicates that the method is numerically exact for idealized functions. Also is illustrated the inherent suppression of the high-frequency components, without using additional smoothing. 
self-deconvolute a smooth function (Fig. 2). Total agreement between the outcome of the self-deconvolution calculation and the initial artificial data was obtained (absolute error per data point $<1.0 \times 10^{-7}$ ). Second, we unfold the same smooth curve with $10 \%$ noise added. The results are shown in Fig. 3. The TDOS from the noise $N(E)$ is slightly distorted, but the main features remain the same. In the bottom curve of Fig. 3 is drawn the difference between the two top curves.
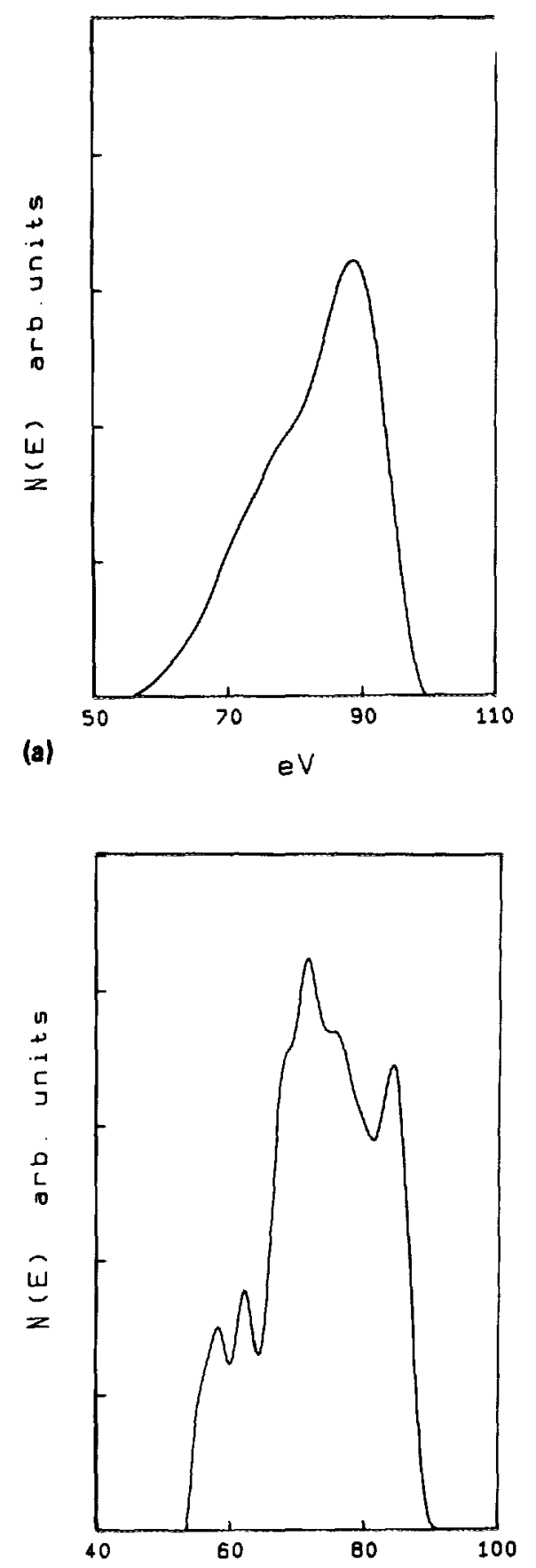

(b)

eV

FIG. 4. The $N(E)$ of the (a) clean and (b) oxidized $\mathrm{Si}(100)$ surface. These two curves are the input for the self-deconvolution calculation. The manipulation of the $L_{23} V V$ Auger spectra to obtain the unbroadened Auger intensity $N(E)$ is discussed elsewhere (Ref. 23).

\section{J. Vac. Sel. Technol. A, Vol. 6, No. 3, May/Jun 1988}

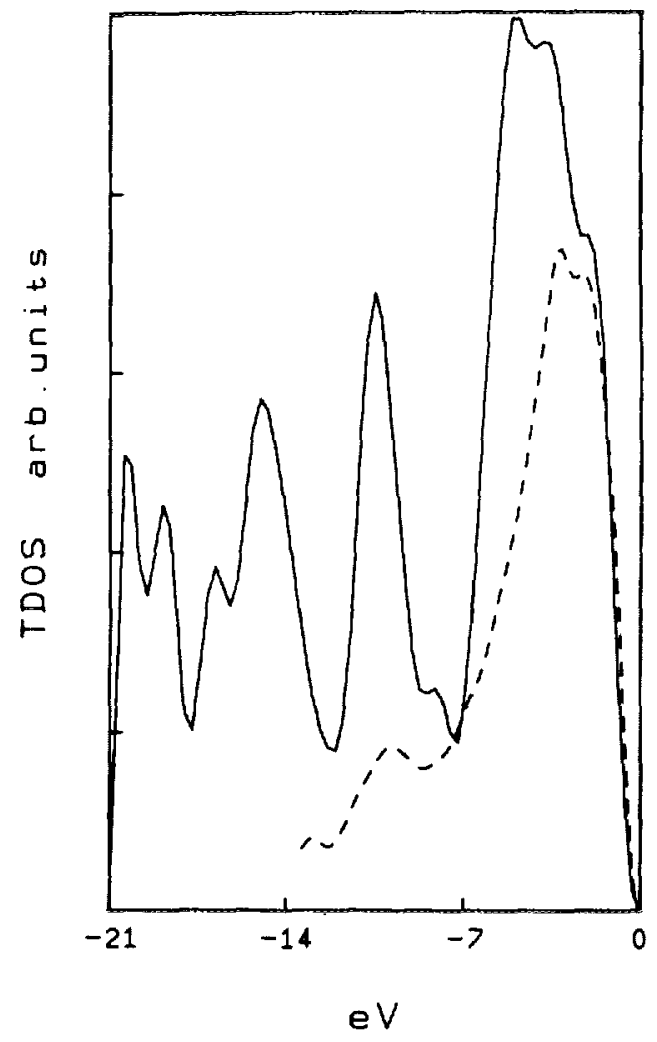

Fig. 5. The TDOS of the --- clean $\mathrm{Si}(100)$ surface, and - of the $\mathrm{SiO}_{2}$ interface. Some characteristic calculation parameters are $k=3, c_{i}=0.2$, $\lambda_{0}=100, N_{\mathrm{s}}=25, N=50$, data points $=200, N_{r}=0$. Residue of $\mathrm{SiO}_{2}<0.1$, and residue of clean $\mathrm{Si}<0.042$. The TDOS of $\mathrm{SiO}_{2}$ cannot be interpreted straightforwardly, because we see also components of $\mathrm{SiO}_{x}$ which can be caused by interface defects or electron beam damage. Also is a matter of debate the nature of the density of state probed by the $L_{2,3} V V$ Auger process.

Our newly developed self-deconvolution method has been applied to the $N(E)$ spectrum of the clean and oxidized $\mathrm{Si}(100)$ surface, as derived from measured $\mathrm{Si}-L_{2,3} V V$ spectra. The experimental setup, surface cleaning procedure, and gas handling have been described in detail elsewhere. ${ }^{21,22}$ The $N(E)$ spectrum can be derived from the Si- $L_{2,3} V V$ spectrum by subtracting the secondary background, followed by a deconvolution of the energy losses and instrumental broadening. ${ }^{23}$ (See Fig. 4.) Preliminary results of the data processing are shown in Fig. 5. The curves in Fig. 5 show good agreement with theoretical calculations of the $\mathrm{SiO}_{x}$ interface by Ching, ${ }^{24}$ and ultraviolet photoemission spectroscopy measurements of Azizan et al. ${ }^{25}$

The interpretation of the spectra will be discussed elsewhere, ${ }^{26}$ but some remarks need to be made about the interpretation of the TDOS. The TDOS cannot be interpreted straightforwardly and certainly not the TDOS of $\mathrm{SiO}_{2}$, because we see also components of $\mathrm{SiO}_{x}$, which may be caused by interface defects or electron beam damage. Also a matter of debate is the nature of the density of state probed by $L_{2,3} V V$ Auger process. ${ }^{2,3,10}$

The method can be applied, in principle, to all problems of the form $A \mathrm{x}=\mathrm{b} \cdot{ }^{23} A$ is a well-defined operation and the vector $b$ is a known array of data points. The vector $\mathbf{x}$ needs to be solved. 
In summary, we have shown that the LM method, used in conjunction with the global approximation method is a powerful tool in obtaining the TDOS of Si from the measured Auger intensity $N(E)$ with a high degree of accuracy. Moreover, the applicability of this method is not restricted only to self-deconvolution in $C V V$ Auger electron spectroscopies and can be considered as a general calculation method.

P. H. Holloway, Adv. Electron. Electron Phys. 54. 241 (1980)

D. E. Ramaker, in Chemistry and Physics of Solid Surfaces $I V$, Vol. 20 in Springer Series in (hemical Physics, edited by R. Vanselow and R. Howe (Springer, Berlin, 1982), p. 19.

'P. J. Feibelman, E. J. McGuire, and K. C. Pandey, Phys. Rev. B 5, 2202 (1977).

D. E. Ramaker, J. S. Murday, N. H. Turner, C. Moore, M. G. Lagally, and

J. E. Houston, Phys Rev B 19, 5375 (1979).

J. J. Lander, Phys. Rev. 91, 1382 (1953)

H. D. Hagstrum and G. E. Becker, Phys. Rev. B 4, 4187 (1971).

H. D. Hagstrum and G. E. Becker, Phys. Rev. B 8, 1580 (1973).

"G. F. Amelio, Surf. Sci. 22, 301 (1970).

"J. E. Houston, J. Vac. Sci. Technol. 12, 255 (1974)

"'R. Weissman and K. Muller, Surf. Sci. Rep. 1, 251 ( 1981 ).

'D. E. Ramaker, Appl. Surf. Sci. 21, 243 (1985).

1'W. M. Mularie and W. T. Peria, Surf. Sci. 26, 125 (1971)
${ }^{1.3}$ M. C. Munoz, J. L. Sacedon, and F. Soria, Surf. Sci. 172, 442 (1986).

${ }^{14}$ R. H. Brockman and G. J. Russel, Phys. Rev. B 22, 6302 (1980).

${ }^{15}$ J. A. Taggle, V. Martinez, J. M. Rojo, and M. Salmeron, Surf. Sci. 79, 93 (1978)

'tV. Martinez, J. Electron Spectrosc. Relat. Phenom. 17, 33 (1979)

17D. E. Ramaker, F. L. Hutson, H. H. Turner, and W. N. Mei, Phys. Rev. B 33, 2574 (1986); D. R. Jennison, ibid. 18, 6865 (1978).

${ }^{1 \times}$ J. H. Onsgaard, P. Morgen, and R. P. Creaser, J. Vac. Sci. Technol. 15, 44 (1978).

${ }^{19} \mathrm{R}$. Fletcher, "A modified Marquardt subroutine for nonlinear leastsquares fitting," U. K. Atomic Energy authority Research Group. Theoretical Physics Division, Report No. AERE-R. 6799, Harwell, Berkshire, United Kingdom, 1971.

${ }^{20}$ P. M. Prenter, Splines and Variational Methods (Wiley, New York, 1975)

"'A. G. B. M. Sasse, P. M. Kleinherenbrink, and A. van Silfhout, Surf. Sci. (in press).

${ }^{22}$ E. G. Keim, L. Wolterbeek, and A. van Silfhout, Surf. Sci. 180, 565 (1987).

${ }^{23}$ A. G. B. M. Sasse, H. Wormeester, and A. van Silfhout, Appl. Surf. Sci (submitted).

${ }^{24}$ W. Y. Ching, Phys. Rev. B 26, 6610, 6622, 6633 (1982)

${ }^{29}$ M. Azizan, R. Baptist, A. Brenac, G. Chauvet, and T. A. Nguyen Tan, J. Phys. (Paris) 48, 81 (1987).

${ }^{26}$ A. G. B. M. Sasse, D. G. Lakerveld, H. Wormeester, and A. van Silfhout (to be published). 